\title{
SINGULAR PERTURBATION ANALYSIS OF THE SCATTERING OF RAYLEIGH WAVES BY THIN SURFACE LAYERS*
}

BY

\author{
D. A. SIMONS
}

Brown University

Introduction. Surface waves progagating in elastic or piezoelectric half-spaces will be scattered upon interaction with thinly plated, finite or semi-infinite surface regions. At large distances from the scatterer along the surface, reflected and transmitted surface waves will appear. The remainder of the field after subtraction of all the surface waves is known as the radiated wave. In plane strain, the surface waves decay exponentially with depth but do not decay with distance along the surface, while the radiated wave decays as $r^{-1 / 2}$ along any ray, and as $r^{-3 / 2}$ along the surface. The power flux associated with the incident wave will be partitioned among the reflected, transmitted, and radiated waves.

Numerous analyses of such interactions have appeared [1-7]. A common feature among all of these is the representation of the surface layer by a set of boundary conditions [8] to be applied to fields in the substrate. (These boundary conditions are accurate to within terms of order (layer thickness)/(wavelength).) The resulting boundary value problems have been solved by various methods. The Wiener-Hopf technique is useful in some very special cases with semi-infinite layers [1-3] but even here usually leads to integrals which can be evaluated only numerically. A variational approach [4-5] and various related modal expansion techniques [6] are more widely applicable and have proven successful for many applications, but have not given any insight into the radiated wave, as they incorporate an assumed field consisting only of surface waves. A direct, approximate, numerical solution has been obtained for a single strip [7], but the numerical analysis is exceedingly complex. Nevertheless, that study suggests that the power flux in the radiated wave may in some instances exceed that in the reflected surface wave.

Thus, in spite of this proliferation of existing approaches, it would still seem useful to devise a method which is capable of treating finite and semi-infinite layers, elastic and piezoelectric substrates, inertial and stiffening effects of the layer, plane and non-plane problems; which accounts for body waves as well as surface waves, and which leads to simple expressions for the scattered field. The purpose of this paper is to present, by way of example, a singular perturbation approach to this class of problems-an approach which appears to possess the attributes listed above. The problem treated in detail is the scattering of a Rayleigh wave by a finite, thin strip. In order not to obscure the fundamental concepts, the density of the layer is taken to vanish, but this restriction is not essential to the success of the method. The problem is formulated in terms of a singular integral equation governing the shearing traction between the strip and the substrate. A parameter

* Received August 17, 1976. The author wishes to express his appreciation to Lawrence Sirovich and George Zahalak for useful discussions regarding the singular perturbation scheme. This work was supported by the National Science Foundation under grant No. ENG75-10554. 
$\epsilon$, proportional to the ratio of layer thickness to incident wavelength, appears explicitly, and an expansion of the solution about $\epsilon=0$ is sought. Boundary layers near the edges of the strip are identified, and inner and outer expansions are postulated in the usual way. Closed-form expressions are obtained for the first term of the outer expansion, and for certain integrals of the inner expansion. These in turn are sufficient to determine closedform expressions for the transmitted and reflected surface waves accurate to order $\epsilon$.

Formulation of the problem. Consider an isotropic elastic half-space $-\infty<(x, y)<$ $\infty, z>0$, with Lamé parameters $\lambda$ and $\mu$ and density $\rho$. A strip of thickness $2 h^{\prime}$ and Lamé parameters $\lambda^{\prime}, \mu^{\prime}$ is bonded to the surface $z=0,0<x<l,-\infty<y<\infty$. The density $\rho^{\prime}$ of the strip is assumed to vanish. Plane strain is assumed to obtain, i.e., the displacement in the $y$-direction vanishes and all field quantities depend only on $x$ and $z$. Only harmonic waves with time dependence $\exp (-i \omega t), \omega>0$, are considered; hereafter the time-factor is dropped and fields are in general taken to be complex.

The boundary conditions are $[4,8]$

$$
\begin{gathered}
\sigma_{x z}(x, 0)=\sigma_{z z}(x, 0)=0 \quad(-\infty<x<0, l<x<\infty), \\
\sigma_{x z}(x, 0)+\left(\mu \epsilon / k_{s}\right) u_{x, x x}(x, 0)=\sigma_{z z}(x, 0)=0 \quad(0<x<l), \\
u_{x, x}\left(0^{+}, 0\right)=u_{x, x}\left(l^{-}, 0\right)=0,
\end{gathered}
$$

where $\sigma_{i j}(x, z)$ are the components of Cauchy stress, $u_{x}(x, z)$ is the $x$-displacement, $\epsilon=$ $8 h^{\prime} k_{s}\left(\mu^{\prime} / \mu\right)\left(\lambda^{\prime}+\mu^{\prime}\right) /\left(\lambda^{\prime}+2 \mu^{\prime}\right), k_{s}=\omega / c_{s}, c_{s}=(\mu / \rho)^{1 / 2}$, and a subscript comma denotes differentiation. Eqs. (1) represent the vanishing of surface traction on the unplated surface of the half-space; Eqs. (2), the interaction of the strip with the half-space; and Eqs. (3), the vanishing of traction on the edges of the strip.

The surface $x$-displacement in the incident Rayleigh wave is

$$
u_{x}^{(i)}(x, 0)=u_{0} \exp \left(i k_{R} x\right)
$$

where $k_{R}$ is the positive root of the equation

$$
R(\kappa) \equiv\left(2 \kappa^{2}-k_{s}^{2}\right)^{2}-4 \kappa^{2} n_{s}(\kappa) n_{d}(\kappa)=0 .
$$

Here $n_{\gamma}(\kappa)=\left(\kappa^{2}-k_{\gamma}{ }^{2}\right)^{1 / 2}, \gamma=d, s ; k_{d}=\omega / c_{d}, c_{d}=[(\lambda+2 \mu) / \rho]^{1 / 2}$. The wave numbers and phase velocities $k_{s}, c_{s}, k_{d}, c_{d}$ are appropriate to plane shear and dilatational waves in unbounded elastic media; the Rayleigh wave propagates along the surface in the positive $x$-direction with speed $c_{R} \equiv \omega / k_{R}\left(<c_{s}<c_{d}\right)$ while decaying exponentially with depth [9]. The associated tractions vanish on the surface $z=0$, i.e.,

$$
\sigma_{x z}{ }^{(i)}(x, 0)=\sigma_{z z}{ }^{(i)}(x, 0)=0 .
$$

Now let the scattered field be defined as the difference between the total field and the incident wave, and denoted with a superscript $(s)$. If $t_{x}(x)$ is the shear traction between the strip and the substrate, then in view of Eq. (6),

$$
t_{x}(x) \equiv \sigma_{x z}(x, 0)=\sigma_{x z}^{(s)}(x, 0) .
$$

Eqs. (1-4, 6-7) then lead to the following boundary conditions for the scattered field:

$$
\begin{gathered}
\sigma_{x z}{ }^{(s)}(x, 0)=\sigma_{z z}{ }^{(s)}(x, 0)=0 \quad(-\infty<x<0, l<x<\infty), \\
t_{x}(x)+\left(\mu \epsilon / k_{s}\right) u_{x, x x}{ }^{(s)}(x, 0)-u_{0} k_{R}{ }^{2}\left(\mu \epsilon / k_{s}\right) \exp \left(i k_{R} x\right)=\sigma_{z z}{ }^{(s)}(x, 0)=0(0<x<l),
\end{gathered}
$$




$$
u_{x, x}^{(s)}\left(0^{+}, 0\right)+i k_{R} u_{0}=u_{x, x^{(s)}}\left(l^{-}, 0\right)+i k_{R} u_{0} \exp \left(i k_{R} l\right)=0 .
$$

The displacement $u_{x}{ }^{(s)}(x, 0)$ may be represented by the superposition integral

$$
u_{x}^{(s)}(x, 0)=\int_{0}^{l} t_{x}(\xi) G_{x x}(x-\xi) d \xi \quad(-\infty<x<\infty),
$$

where [10]

$$
G_{x x}(x)=(1 / 2 \pi \mu) \int_{-\infty}^{\infty}\left[k_{s}{ }^{2} n_{s}(\kappa) / R(\kappa)\right] \exp (i \kappa x) d \kappa .
$$

The integration contour in Eq. (12) must be indented (in the complex $k$-plane) above the branch points at $-k_{s},-k_{d}$, and the pole at $-k_{R}$, and below the branch points at $k_{s}, k_{d}$, and the pole at $k_{R}$, and the multivalued functions $n_{s}(\kappa), n_{d}(\kappa)$ taken as positive when $\kappa>$ $k_{s}$.

The Green's function $G_{x x}(x)$ is the surface $x$-displacement due to a surface shearing traction $\delta(x)$ (where $\delta(x)$ is the Dirac delta function), and accordingly, along with its associated $z$-displacement and stresses, satisfies all the field equations of dynamic elasticity and propagates outward from the origin. The latter two facts apply as well to the superposition given by Eq. (11). Further, because the Green's state is free of surface shearing traction away from the source point, and free of normal traction over the entire surface, Eqs. (8) and the second of Eqs. (9) are satisfied automatically so it only remains to determine $t_{x}(x), 0<x<l$, in accord with Eq. (10) and the first of Eqs. (9).

By replacing $x$ by $\xi$ in the first of Eqs. (9), multiplying by $\operatorname{sgn}(x-\xi)$ (where $\operatorname{sgn}(x)=1$ if $x>0,-1$ if $x<0$ ), integrating from 0 to $l$, and employing Eqs. (10-11), there results

$$
\begin{aligned}
\int_{0}^{l} t_{x}(\xi)[\operatorname{sgn}(x-\xi) & \left.+\frac{2 \mu \epsilon}{k_{s}} G_{x x, x}(x-\xi)\right] d \xi \\
= & -\frac{2 \mu \epsilon i k_{R} u_{0}}{k_{s}} \exp \left(i k_{R} x\right) \quad(0<x<l),
\end{aligned}
$$

where $G_{x x, x}(x)$ is obtained from Eq. (12) by dividing the integrand by $i \kappa$. This function will have a Cauchy singularity at the origin, so the integral in Eq. (13) must be interpreted in the sense of Cauchy's principal value. By directly integrating the first of Eqs. (9) from 0 to $l$, and using Eq. (10), there results

$$
\int_{0}^{l} t_{x}(\xi) d \xi=0
$$

Eq. (13) may be regarded as a singular integral equation governing $t_{x}(x)$ and Eq. (14) as an auxiliary condition. Recall that $t_{x}(x)$ is a shear stress, so it may possess integrable singularities of the form $r^{-p}, p<1$ at $x=0^{+}, l^{-}$, without causing the strain energy to become unbounded. Muskhelishvili's general theory [11], when applied to this case, guarantees that Eqs. (13-14) do in fact uniquely determine $t_{x}(x)$.

These and all subsequently appearing equations may be non-dimensionalized by the transformations

$$
\begin{gathered}
\bar{x}=x k_{s} ; \quad \bar{\xi}=\xi k_{s} ; \quad k_{R}=k_{R} / k_{s} ; \quad l=l k_{s} ; \\
\bar{t}_{x}(\bar{x})=t_{x}(x) /\left(\mu u_{0} k_{s}\right) ; \quad \bar{G}_{x x, x}(\bar{x})=\left(\mu / k_{s}\right) G_{x x, x}(x) ; \\
\bar{\kappa}=\kappa / k_{s} ; \quad \bar{n}_{\gamma}(\bar{\kappa})=n_{\gamma}(\kappa) / k_{s}, \quad k_{\gamma}=k_{\gamma} / k_{s}, \quad \gamma=d, s\left(\text { so } k_{s} \equiv 1\right) ; \\
\bar{R}(\bar{\kappa})=R(\kappa) / k_{s}{ }^{4} ; \quad \bar{u}_{x}^{(s)}(\bar{x}, 0)=u_{x}^{(s)}(x, 0) / u_{0},
\end{gathered}
$$


after which the overbars are suppressed for convenience. The dimensionless form of Eqs. $(13-14)$ are thus

$$
\begin{gathered}
\int_{0}^{l} t_{x}(\xi)\left[\operatorname{sgn}(x-\xi)+2 \epsilon G_{x x, x}(x-\xi)\right] d \xi=-2 \epsilon i k_{R} \exp \left(i k_{R} x\right) \quad(0<x<l) \\
\int_{0}^{l} t_{x}(\xi) d \xi=0 .
\end{gathered}
$$

Singular perturbation analysis. The parameter $\epsilon$, defined following Eq. (3), is a product of elastic constants and $h^{\prime} k_{s} \equiv\left(2 h^{\prime} / \lambda_{R}\right)\left(k_{s} / k_{R}\right) \pi$, where $\lambda_{R}=2 \pi / k_{R}$ is the incident wavelength. Because $k_{s} / k_{R}$ also depends only on elastic constants (cf. Eq. (5)), $\epsilon$ is a measure of layer thickness relative to incident wavelength, and in many applications of interest will be extremely small. Furthermore, the representation of the surface layer by boundary condition (2) is accurate only to order $2 h^{\prime} / \lambda_{R}$ [8]. Thus a perturbation analysis of Eqs. (15-16), accurate to order $\epsilon$, is justified.

As with most analyses of this type, a certain amount of trial and error is involved in ascertaining the correct transformations and expansions, so some of the following steps may at first appear unmotivated. However, some reflection will reveal that the transformations and expansions postulated below are the only ones which permit Eqs. (15-16) to be satisfied to order $\epsilon$.

The outer limit process is simply $\epsilon \rightarrow 0$ with $x$ fixed. Boundary layers will exist near both ends of the interval $(0, l)$, corresponding respectively to the inner limit processes $\epsilon \rightarrow 0$ with $\hat{x} \equiv x / \epsilon>0$ fixed; and $\epsilon \rightarrow 0$ with $\tilde{x} \equiv(x-l) / \epsilon<0$ fixed. Thus it is assumed that $t_{x}$ has the following expansions:

$$
\begin{gathered}
t_{x}(x ; \epsilon)=\epsilon t_{1}(x)+o(\epsilon) \quad(\epsilon \rightarrow 0, x \text { fixed }), \\
t_{x}(x ; \epsilon)=\hat{t}_{0}(\hat{x})+o(1) \quad(\epsilon \rightarrow 0, \hat{x} \equiv x / \epsilon \text { fixed }), \\
t_{x}(x ; \epsilon)=\tilde{t}_{0}(\tilde{x})+o(1) \quad(\epsilon \rightarrow 0, \tilde{x} \equiv[x-l] / \epsilon \text { fixed }) .
\end{gathered}
$$

Now suppose the interval of integration $(0, l)$ is partitioned into three subintervals by the points $\epsilon^{1 / 2}, l-\epsilon^{1 / 2}$. Substitution of (17-19) into (15-16), followed by changes of variable $\hat{\xi}=\xi / \epsilon$ in $\left(0, \epsilon^{1 / 2}\right)$, and $\xi=(\xi-l) / \epsilon$ in $\left(l-\epsilon^{1 / 2}, l\right)$, yields

$$
\begin{aligned}
& \epsilon \int_{0}^{\epsilon^{-1 / 2}}\left[\hat{t}_{0}(\xi)+o(1)\right]\left[\operatorname{sgn}(\hat{x}-\xi)+\beta /(\hat{x}-\xi)+2 \epsilon G^{\prime}(\epsilon \hat{x}-\epsilon \xi)\right] d \xi \\
& +\int_{\epsilon^{-1 / 2}}^{l-\epsilon}\left[\epsilon t_{1}(\xi)+o(\epsilon)\right]\left[\operatorname{sgn}(x-\xi)+\epsilon \beta /(x-\xi)+2 \epsilon G^{\prime}(x-\xi)\right] d \xi \\
& +\epsilon \int_{-\epsilon_{-1 / 2}}^{0}\left[\tilde{t}_{0}(\xi)+o(1)\right]\left[\operatorname{sgn}(\tilde{x}-\tilde{\xi})+\beta /(\tilde{x}-\tilde{\xi})+2 \epsilon G^{\prime}(\epsilon \tilde{x}-\epsilon \tilde{\xi})\right] d \tilde{\xi} \\
& =-2 \epsilon i k_{R} \exp \left(i k_{R} x\right) \quad(0<x<l), \\
& \epsilon \int_{0}^{\epsilon^{1 / 2}}\left[\hat{t}_{0}(\xi)+o(1)\right] d \xi+\int_{\epsilon^{1 / 2}}^{l-\epsilon^{1 / 2}}\left[\epsilon t_{1}(\xi)+o(\epsilon)\right] d \xi \\
& +\epsilon \int_{-\epsilon^{-1 / 2}}^{0}\left[\tilde{t}_{0}(\xi)+o(1)\right] d \xi=0,
\end{aligned}
$$


where $\hat{x} \equiv x / \epsilon, \tilde{x} \equiv(x-l) / \epsilon, \beta=1 /\left[\pi\left(1-k_{d}{ }^{2} / k_{s}{ }^{2}\right)\right]$, and $G^{\prime}(x)=G_{x x, x}(x)-\beta / 2 x$. The Cauchy singularity has been separated out of the kernel $G_{x x, x}(x)$ by performing an asymptotic analysis of this function (as defined by the derivative of Eq. (12)) in the vicinity of $x=0$. The remainder, denoted $G^{\prime}(x)$, is bounded for $-\infty<x<\infty$.

The outer solution, reflection and transmission coefficients. In the outer limit $x$ is fixed, $\epsilon \rightarrow 0, \hat{x} \rightarrow \infty$, and $\tilde{x} \rightarrow-\infty$. After dividing by $\epsilon$ and performing the outer limit process, Eqs. (20-21) yield

$$
\begin{gathered}
\tilde{I}_{0}+\int_{0}^{l} t_{1}(\xi) \operatorname{sgn}(x-\xi) d \xi-\tilde{I}_{0}=-2 i k_{R} \exp \left(i k_{R} x\right) \quad(0<x<l), \\
\tilde{I}_{0}+\int_{0}^{l} t_{1}(\xi) d \xi+\tilde{I}_{0}=0
\end{gathered}
$$

where

$$
\tilde{I}_{0} \equiv \int_{0}^{\infty} \hat{t}_{0}(\xi) d \xi, \quad \tilde{I}_{0} \equiv \int_{-\infty}^{0} \tau_{0}(\xi) d \xi .
$$

Eq. (22) is solved simply by differentiating it with respect to $x$, yielding

$$
t_{1}(x)=k_{R}^{2} \exp \left(i k_{R} x\right) \quad(0<x<l) .
$$

After substitution of (25) into (22-23), the latter two equations may be solved simultaneously for $\hat{I}_{0}$ and $\tilde{I}_{0}$, yielding

$$
I_{0}=-i k_{R}, \quad I_{0}=i k_{R} \exp \left(i k_{R} l\right) .
$$

Thus it has been possible to determine the outer expansion of $t_{x}(x)$ to order $\epsilon$, as well as the integrals $I_{0}, I_{0}$ of the zero-order inner expansions, by applying the outer limit process to the governing singular integral equation (15) and the auxiliary condition (16). This information alone will be sufficient to determine, through the superposition formula (11), the complex amplitudes of the reflected and transmitted surface waves. Substitution of (12) into (11), interchange of the sequence of integrations, and non-dimensionalization as before, yield

$$
u_{x}^{(s)}(x, 0)=\frac{1}{2 \pi} \int_{-\infty}^{\infty} \frac{n_{s}(\kappa)}{R(\kappa)} \exp (i \kappa x)\left[\int_{0}^{l} t_{x}(\xi) \exp (-i \kappa \xi) d \xi\right] d \kappa \quad(-\infty<x<\infty) .
$$

For $x<0$ the outer integration contour in Eq. (27) may be closed by large arcs in the lower half $\kappa$-plane and loops about the branch cuts emanating into the third quadrant from $\kappa=-k_{s}$ and $-k_{d}$. Recall that the original contour was indented above the pole at $\kappa=$ $-k_{R}$, so application of Jordan's Lemma and the residue theorem yields

$$
\begin{aligned}
u_{x}^{(s)}(x, 0)=-\frac{i n_{s}\left(-k_{R}\right)}{\left.(d R / d \kappa)\right|_{\kappa=-k_{R}}} \exp \left(-i k_{R} x\right) \quad \int_{0}^{l} t_{x}(\xi) \exp \left(i k_{R} \xi\right) d \xi & \\
& + \text { B.L.I. } \quad(-\infty<x<0),
\end{aligned}
$$

where B.L.I. denotes the branch line integrals. These may be shown by Watson's Lemma to decay as $|x|^{-3 / 2}$ as $x \rightarrow-\infty$, so the first term of the right-hand side of Eq. (28) represents the reflected surface wave, a negatively propagating Rayleigh wave. Its complex amplitude (i.e., the coefficient of $\exp \left(-i k_{R} x\right)$ ), is denoted $A_{r}$ and referred to as the reflection 
coefficient. The integral appearing in Eq. (28) may be evaluated by the same partitioning and expansions as were employed previously, i.e.,

$$
\begin{aligned}
\int_{0}^{l} t_{x}(\xi) \exp \left(i k_{R} \xi\right) d \xi=\epsilon \int_{0}^{\epsilon-1 / 2}\left[\hat{t_{0}}(\hat{\xi})+o(1)\right] \exp \left(i k_{R} \epsilon \hat{\xi}\right) d \hat{\xi} \\
+\int_{\epsilon^{1 / 2}}^{l-\epsilon^{1 / 2}}\left[\epsilon t_{1}(\xi)+o(\epsilon)\right] \exp \left(i k_{R} \xi\right) d \xi+ \\
\epsilon \int_{-\epsilon^{-1 / 2}}^{0}\left[\tilde{t_{0}}(\tilde{\xi})+o(1)\right] \exp \left(i k_{R}(\epsilon \tilde{\xi}+l)\right) d \tilde{\xi}
\end{aligned}
$$

Substitution of Eqs. (24-26) into (29) yields

$$
\begin{aligned}
\int_{0}^{l} t_{x}(\xi) \exp \left(i k_{R} \xi\right) d \xi & =\epsilon\left[\hat{I}_{0}-\left(i k_{R} / 2\right)\left(\exp \left(2 i k_{R} l\right)-1\right)+\exp \left(i k_{R} l\right) \tilde{I}_{0}\right]+o(\epsilon) \\
& =\epsilon\left(i k_{R} / 2\right)\left(\exp \left(2 i k_{R} l\right)-1\right)+o(\epsilon)
\end{aligned}
$$

so from Eqs. $(28,30)$ the reflection coefficient is

$$
A_{r}=-\frac{i n_{s}\left(-k_{R}\right)}{\left.(d R / d \kappa)\right|_{\kappa=-k_{R}}}\left[\epsilon\left(i k_{R} / 2\right)\left(\exp \left(2 i k_{R} l\right)-1\right)+o(\epsilon)\right] .
$$

When $x>l$, the integration contour in Eq. (27) may be closed in the upper half $\kappa$ plane. The contribution from the pole at $k_{R}$ is a positively propagating Rayleigh wave and may be evaluated analogously. When this is combined with the incident wave, the transmission coefficient $A_{t}$, defined as the coefficient of $\exp \left(i k_{R} x\right)$, is found to be

$$
A_{t}=1+\frac{i k_{R} \ln _{s}\left(k_{R}\right)}{\left.(d R / d \kappa)\right|_{\kappa=k_{R}}} \epsilon+o(\epsilon) \text {. }
$$

The inner solution. By employing the subsurface fields of the Green's state in formulas analogous to Eq. (11), it would now be possible to evaluate the scattered field at any subsurface point of the half-space, accurate to order $\epsilon$. The inner expansions need not be determined explicitly for this purpose, as only the integrals $\hat{I}_{0}, \tilde{I}_{0}$ would appear, and these have already been determined. However, a study of the inner expansions will elicit the form and strength of the singularity in the shear traction $t_{x}(x)$ near the edges of the strip. Further, an interesting parallel will be seen to exist between the inner expansion and a related static problem.

Consider then, for example, an application of the left-hand inner limit process to Eq. (20). In this limit $\hat{x}=x / \epsilon$ is fixed, $\epsilon \rightarrow 0, x \rightarrow 0$, and $\tilde{x} \rightarrow-\infty$. Note that Eqs. $(24,26)$ imply

$$
\int_{0}^{\infty} \hat{t_{0}}(\hat{\xi}) d \hat{\xi}=-i k_{R}
$$

After division by $\epsilon$, application of the left-hand inner limit process, substitution from Eq. (33), and some rearrangement, Eq. (20) yields

$$
\int_{0}^{\hat{x}} \hat{t}_{0}(\xi) d \hat{\xi}+(\beta / 2) \int_{0}^{\infty}\left[\hat{t}_{0}(\hat{\xi}) /(\hat{x}-\hat{\xi})\right] d \hat{\xi}=-i k_{R} \quad(0<\hat{x}<\infty) .
$$

Eqs. (33-34) are very similar to those obtained by Koiter [11] governing static load 
transfer from a semi-infinite edge stiffener into a sheet in plane stress. Indeed, the transformations

$$
\begin{gathered}
\eta=4 \hat{\xi}\left(1-{k_{d}}^{2} /{k_{s}}^{2}\right), \quad v=4 \hat{x}\left(1-{k_{d}}^{2} /{k_{s}}^{2}\right), \\
\tau(\eta)=\hat{t}_{0}(\hat{\xi}) /\left[-4 i k_{R}\left(1-{k_{d}}^{2} /{k_{s}}^{2}\right)\right],
\end{gathered}
$$

when used in Eqs. (33-34), yield

$$
\begin{gathered}
\int_{0}^{v} \tau(\eta) d \eta-(2 / \pi) \int_{0}^{\infty}[\tau(\eta) /(\eta-v)] d \eta-1=0 \quad(0<v<\infty), \\
\int_{0}^{\infty} \tau(\eta) d \eta=1
\end{gathered}
$$

which are precisely the equations solved by Koiter. He presents the following asymptotic results:

$$
\begin{gathered}
P(v) / P_{0} \sim 1-(2 v / \pi)^{1 / 2} \text { as } v \rightarrow 0^{+}, \\
P(v) / P_{0} \sim 2 /(\pi v) \text { as } v \rightarrow \infty,
\end{gathered}
$$

where

$$
P(v) / P_{0}=\int_{v}^{\infty} \tau(\eta) d \eta
$$

Eq. (40) shows that $\tau(v)=-d\left[P / P_{0}\right] / d v$, so (38-39) imply

$$
\begin{gathered}
\tau(v) \sim(2 \pi v)^{-1 / 2} \text { as } v \rightarrow 0^{+}, \\
\tau(v) \sim 2 /\left(\pi v^{2}\right) \text { as } v \rightarrow \infty .
\end{gathered}
$$

Substitution from (35) into (41) yields the singular form of the traction near the edge of the strip:

$$
t_{0}(\hat{x})=-2 i k_{R}\left[\left(1-k_{d}{ }^{2} / k_{s}{ }^{2}\right) / 2 \pi \hat{x}\right]^{1 / 2} \text { as } \hat{x} \rightarrow 0 .
$$

When Eq. (43) is written in terms of the outer variable $x=\epsilon \hat{x}$, it is seen that the singularity is of inverse square root type in $x$, with strength proportional to $\epsilon^{1 / 2}$.

Analysis of the right-hand boundary layer would follow the same steps as those employed here, and would lead to similar results.

Discussion. Several questions naturally arise with regard to the preceding analysis and results. First, how could the lowest terms of both the inner and outer expansions of $t_{x}$ have been fully determined without recourse to any matching principle (e.g., [12])? Furthermore, Eqs. $(17,25)$ show that the one-term, left-hand inner expansion of the oneterm outer expansion $\epsilon t_{1}(x)$ is just $\epsilon k_{R}{ }^{2}$, while Eqs. $(18,35,42)$ show that the one-term outer expansion of the one-term, left-hand inner expansion $\hat{t}_{0}(\hat{x})$ is proportional to $\epsilon^{2} / x^{2}$. How can the solution be correct in view of this apparent contradiction of Van Dyke's matching principle?

In answer to the first, it should be noted that the necessity of applying a matching principle arises when problems are formulated in terms of differential equations which are solved successively in various adjacent, overlapping domains. Because the present approach begins with an integral equation whose domain spans both the inner and outer regions, such a need does not arise. 
To answer satisfactorily the second question, or to generalize the circumstances under which matching need not apply, would require a fuller theoretical basis than is now available. It may be noted, however, that the matching principle is satisfied by the stresses in the interior of the two-dimensional domain $-\infty<x<\infty, 0<z<\infty$, and by the displacements in the closure of this domain. (An example will be given presently.) Among these field quantities it is only the shear stress $\sigma_{x z}$ which fails to satisfy the matching principle, and it is only on the layer-substrate interface, near the edges, that it does so. But this is precisely the surface on which the non-classical boundary condition (9) and the related edge condition (10) obtain. The fact that (9) enforces a linear relationship between $t_{x}$ and the second derivative of a displacement is undoubtedly behind the failure of the derived expansions of $t_{x}$ to satisfy the matching condition.

Now it will be shown that the inner and outer expansions of $u_{x}{ }^{(s)}$ do satisfy the matching condition. (Similar manipulations would confirm the matching of the vertical displacement and the stresses in the regions just mentioned.) When the subdivision and variable changes of (20-21) are used in (11), and $G_{x x}(x-\xi)$ is replaced by its subsurface extension $G_{x x}(x-\xi, z)$, there results

$$
\begin{aligned}
u_{x}^{i s}(x, z)= & \epsilon \int_{0}^{\epsilon^{-1 / 2}}\left[\hat{t}_{0}(\hat{\xi})+o(1)\right] G_{x x}(x-\epsilon \hat{\xi}, z) d \hat{\xi} \\
& +\int_{\epsilon^{1 / 2}}^{l-\epsilon^{-1 / 2}}\left[\epsilon t_{1}(\xi)+o(\epsilon)\right] G_{x x}(x-\xi, z) d \xi \\
& +\epsilon \int_{-\epsilon^{-1 / 2}}^{0}\left[\tilde{t_{0}}(\xi)+o(1)\right] G_{x x}(x-l-\epsilon \xi, z) d \xi
\end{aligned}
$$

Application of the outer limit process to (44), and substitution from (24), yield

$$
\begin{gathered}
u_{x}^{\prime s}(x, z) \sim \epsilon\left[\hat{I}_{0} G_{x x}(x, z)+\int_{0}^{l} t_{1}(\xi) G_{x x}(x-\xi, z) d \xi\right. \\
\left.+\tilde{I}_{0} G_{x x}(x-l, z)\right] \quad(\epsilon \rightarrow 0, \quad x, z \text { fixed }) .
\end{gathered}
$$

This is the one-term outer expansion of $u_{x}^{(s)}$ and consists of line-load fields of strength $\epsilon \hat{I}_{0}, \epsilon \tilde{I}_{0}$ emanating respectively from $x=0, z=0$ and $x=l, z=0$, combined with a superposition integral. Its one-term left-hand inner expansion is obtained by letting $\epsilon \rightarrow 0$ with $\hat{x}=x / \epsilon, \hat{z}=z / \epsilon$ fixed. Due to the logarithmic singularity at the origin of the line-load horizontal displacement field, only the first term of (45) contributes, giving

$$
u_{x}^{(s)}(x, z) \sim \hat{I}_{0}(\beta / 2) \epsilon \log \epsilon .
$$

The one-term left-hand inner expansion of $u_{x}^{(s)}$ results from application of the lefthand inner limit process to (44). Again the first term dominates, and the limit process yields an expression identical to (46). The outer expansion of this clearly satisfies the matching principle.

It should be noted from the foregoing that both the left- and right-hand inner expansions of $t_{x}$ as well as the outer expansion contribute to the outer expansion of $u_{x}^{(s)}$, while only $\hat{t}_{0}$, through $\hat{I}_{0}$, enters the lowest order term of the left-hand inner expansion of $u_{x}^{(s)}$. In other words, it is not true that the three terms on the right-hand side of (44) determine respectively the lowest order left-hand, outer, and right-hand expansions of $u_{x}^{(s)}$. 
If the individual contributions of the three terms of (45) to the reflection coefficient $A_{r}$ are computed, they are found to be proportional respectively to the three terms in (30). It is curious to note that the first and third of these, which come from the boundary layers, serve only to reverse the sign of the second, which comes from the outer region.

The reflection coefficient $A_{r s}$ for a semi-infinite surface layer (covering $0<x<\infty$ ) may easily be inferred from Eq. (31) to first order. The reflection from the strip may be considered as a sum of independent reflections from the two edges at $x=0$ and $x=l$. The portion of $A_{r}$ which is independent of $l$ then must represent the reflection from the leading edge (at $x=0)$, so from Eq. (31),

$$
A_{r s}=-\frac{\epsilon k_{R} n_{s}\left(-k_{R}\right)}{\left.2(d R / d \kappa)\right|_{\kappa=-k_{R}}}+o(\epsilon) .
$$

This formula may be checked with an existing solution for reflection by a semi-infinite layer obtained by the Wiener-Hopf technique [3]. That analysis employed the same boundary condition as was used here, but obtained a solution of the resulting equations valid for arbitrary $\epsilon$. When it is noted that the reflection coefficient $A_{r}$ for $x$-displacement is the negative of the reflection coefficient for the potential used in [3], the results may be shown to agree to order $\epsilon$ as $\epsilon \rightarrow 0$.

When a similar check is made of the result of Yoneyama and Nishida [12] for the same problem, it may be shown that when the layer's density vanishes, their formula for $A_{r}$ differs in sign from the present one. That analysis seems to take no explicit account of the edge condition [the first of Eqs. (3)], and in view of the earlier comments regarding the contributions of the inner expansions to $A_{r}$, the discrepancy is not surprising.

\section{REFERENCES}

[1] D. Quak and F. L. Neerhoff, Reflection, transmission, and excitation of SH-surface waves by a discontinuity in mass-loading on a semi-infinite elastic medium, Appl. Sci. Res. 29, 447-460 (1974)

[2] D. A. Simons, Scattering of a Love wave by the edge of a thin surface layer. J. Appl. Mech. 42, 842-846 (1975)

[3] D. A. Simons, Scattering of a Rayleigh wave by the edge of a thin surface layer of negligible inertia, J. Acoust. Soc. Am. 59, 12-18 (1976)

[4] B. K. Sinha and H. F. Tiersten, Elastic and prezoelectric surface waves guided by thin films, J. Appl. Phys. 44, 4831-4854 (1973)

[5] B. K. Sinha and H. F. Tiersten, A variational analysis of the reflection of surface waves by arrays of reflecting strips, J. Appl. Phys. 47, 2824-2832 (1976)

[6] B. A. Auld, Acoustic fields and waves in solids, Wiley, New York, 1973

[7] D. A. Simons, Scattering of normally incident Rayleigh waves by thin strips, J. Acoust. Soc. Am. 60, 1100-1107 (1976)

[8] H. F. Tiersten, Elastic surface waves guided by thin films, J. Appl. Phys. 40, 770-790 (1969)

[9] A. E. H. Love, A treatise on the mathematical theory of elasticity, fourth ed., Dover, New York, 1944

[10] H. Lamb, On the propagation of tremors over the surface of an elastic solid, Phil. Trans. Royal Soc. Lond., Series A, 203, 1-42 (1904)

[11] W. T. Koiter, On the diffusion of load from a stiffener into a sheet, Quart. J. Mech. Appl. Math. 8, 164-178 (1954)

[12] M. Van Dyke, Perturbation methods in fluid mechanics, Academic, New York, 1964

[13] T. Yoneyama and S. Nishida, Reflection and transmission of Rayleigh waves by the edge of a deposited thin film, J. Acoust. Soc. Am. 55, 738-743 (1974) 